\title{
Measurement of the longitudinal spin asymmetries for weak boson production in proton-proton collisions at $\sqrt{s}=510 \mathrm{GeV}$
}

J. Adam, ${ }^{12}$ L. Adamczyk, ${ }^{2}$ J. R. Adams, ${ }^{35}$ J. K. Adkins, ${ }^{26}$ G. Agakishiev, ${ }^{24}$ M. M. Aggarwal,${ }^{37}$ Z. Ahammed,${ }^{57}$ I. Alekseev, ${ }^{3,31}$ D. M. Anderson, ${ }^{51}$ R. Aoyama, ${ }^{54}$ A. Aparin, ${ }^{24}$ D. Arkhipkin, ${ }^{5}$ E. C. Aschenauer, ${ }^{5}$ M. U. Ashraf, ${ }^{53}$ F. Atetalla, ${ }^{25}$ A. Attri, ${ }^{37}$ G. S. Averichev,${ }^{24}$ V. Bairathi, ${ }^{32}$ K. Barish, ${ }^{9}$ A. J. Bassill, ${ }^{9}$ A. Behera, ${ }^{49}$ R. Bellwied,${ }^{19}$ A. Bhasin,${ }^{23}$ A. K. Bhati, ${ }^{37}$ J. Bielcik, ${ }^{13}$ J. Bielcikova, ${ }^{34}$ L. C. Bland, ${ }^{5}$ I. G. Bordyuzhin, ${ }^{3}$ J. D. Brandenburg, ${ }^{5}$ A. V. Brandin, ${ }^{31}$ D. Brown, ${ }^{28}$ J. Bryslawskyj, ${ }^{9}$ I. Bunzarov, ${ }^{24}$ J. Butterworth, ${ }^{42}$ H. Caines,${ }^{60}$ M. Calderón de la Barca Sánchez,${ }^{7}$ D. Cebra, ${ }^{7}$ I. Chakaberia, ${ }^{25,46}$ P. Chaloupka, ${ }^{13}$ B. K. Chan, ${ }^{8}$ F-H. Chang, ${ }^{33}$ Z. Chang, ${ }^{5}$ N. Chankova-Bunzarova ${ }^{24}$ A. Chatterjee, ${ }^{57}$ S. Chattopadhyay, ${ }^{57}$ J. H. Chen, ${ }^{47}$ X. Chen,${ }^{45}$ J. Cheng, ${ }^{53}$ M. Cherney, ${ }^{12}$ W. Christie, ${ }^{5}$ G. Contin, ${ }^{27}$ H. J. Crawford, ${ }^{6}$ M. Csanad, ${ }^{15}$ S. Das, ${ }^{10}$ T. G. Dedovich, ${ }^{24}$ I. M. Deppner, ${ }^{18}$ A. A. Derevschikov, ${ }^{39}$ L. Didenko, ${ }^{5}$ C. Dilks,${ }^{38}$ X. Dong, ${ }^{27}$ J. L. Drachenberg, ${ }^{1}$ J. C. Dunlop, ${ }^{5}$ T. Edmonds, ${ }^{40}$ L. G. Efimov, ${ }^{24}$ N. Elsey,${ }^{59}$ J. Engelage, ${ }^{6}$ G. Eppley, ${ }^{42}$ R. Esha, ${ }^{8}$ S. Esumi ${ }^{54}$ O. Evdokimov, ${ }^{11}$ J. Ewigleben, ${ }^{28}$ O. Eyser ${ }^{5}$ R. Fatemi, ${ }^{26}$ S. Fazio, ${ }^{5}$ P. Federic,${ }^{34}$ J. Fedorisin, ${ }^{24}$ Y. Feng ${ }^{40}$ P. Filip, ${ }^{24}$ E. Finch, ${ }^{48}$ Y. Fisyak,${ }^{5}$ C. E. Flores, ${ }^{7}$ L. Fulek, ${ }^{2}$ C. A. Gagliardi,${ }^{51}$ T. Galatyuk, ${ }^{14}$ F. Geurts ${ }^{42}$ A. Gibson, ${ }^{56}$ D. Grosnick, ${ }^{56}$ D. S. Gunarathne, ${ }^{50}$ A. Gupta, ${ }^{23}$ W. Guryn ${ }^{5}$ A. I. Hamad, ${ }^{25}$ A. Hamed, ${ }^{51}$ A. Harlenderova, ${ }^{13}$ J. W. Harris, ${ }^{60}$ L. He, ${ }^{40}$ S. Heppelmann, ${ }^{7}$ S. Heppelmann, ${ }^{38}$ N. Herrmann, ${ }^{18}$ L. Holub ${ }^{13}$ Y. Hong, ${ }^{27}$ S. Horvat, ${ }^{60}$ B. Huang, ${ }^{11}$ H. Z. Huang, ${ }^{8}$ S. L. Huang, ${ }^{49}$ T. Huang, ${ }^{33}$ X. Huang, ${ }^{53}$ T. J. Humanic, ${ }^{35}$ P. Huo, ${ }^{49}$ G. Igo, ${ }^{8}$ W. W. Jacobs, ${ }^{21}$ A. Jentsch, ${ }^{52}$ J. Jia, ${ }^{5,49}$ K. Jiang, ${ }^{45}$ S. Jowzaee, ${ }^{59}$ X. Ju, ${ }^{45}$ E. G. Judd ${ }^{6}$ S. Kabana, ${ }^{25}$ S. Kagamaster, ${ }^{28}$ D. Kalinkin, ${ }^{21}$ K. Kang, ${ }^{53}$ D. Kapukchyan, ${ }^{9}$ K. Kauder, ${ }^{5}$ H. W. Ke, ${ }^{5}$ D. Keane, ${ }^{25}$ A. Kechechyan, ${ }^{24}$ M. Kelsey,${ }^{27}$ D. P. Kikoła ${ }^{58}$ C. Kim,${ }^{9}$ T. A. Kinghorn, ${ }^{7}$ I. Kisel, ${ }^{16}$ A. Kisiel ${ }^{58}$ M. Kocan,${ }^{13}$ L. Kochenda,${ }^{31}$ L. K. Kosarzewski, ${ }^{13}$ A. F. Kraishan ${ }^{50}$ L. Kramarik, ${ }^{13}$ P. Kravtsov, ${ }^{31}$ K. Krueger, ${ }^{4}$ N. Kulathunga Mudiyanselage, ${ }^{19}$ L. Kumar, ${ }^{37}$ R. Kunnawalkam Elayavalli, ${ }^{59}$ J. Kvapil, ${ }^{13}$ J. H. Kwasizur, ${ }^{21}$ R. Lacey, ${ }^{49}$ J. M. Landgraf, ${ }^{5}$ J. Lauret, ${ }^{5}$ A. Lebedev, ${ }^{5}$ R. Lednicky, ${ }^{24}$ J. H. Lee, ${ }^{5}$ C. Li,${ }^{45}$ W. Li ${ }^{42}$ W. Li, ${ }^{47}$ X. Li, ${ }^{45}$ Y. Li,${ }^{53}$ Y. Liang,${ }^{25}$ R. Licenik, ${ }^{13}$ J. Lidrych, ${ }^{13}$ T. Lin, ${ }^{51}$ A. Lipiec,${ }^{58}$ M. A. Lisa,${ }^{35}$ F. Liu, ${ }^{10}$ H. Liu, ${ }^{21}$ P. Liu, ${ }^{49}$ P. Liu, ${ }^{47}$ X. Liu, ${ }^{35}$ Y. Liu,${ }^{51}$ Z. Liu, ${ }^{45}$ T. Ljubicic, ${ }^{5}$ W. J. Llope,${ }^{59}$ M. Lomnitz ${ }^{27}$ R. S. Longacre, ${ }^{5}$ S. Luo ${ }^{11}$ X. Luo, ${ }^{10}$ G. L. Ma, ${ }^{47}$ L. Ma, ${ }^{17}$ R. Ma, ${ }^{5}$ Y. G. Ma,${ }^{47}$ N. Magdy,${ }^{49}$ R. Majka,${ }^{60}$ D. Mallick, ${ }^{32}$ S. Margetis, ${ }^{25}$ C. Markert ${ }^{52}$ H. S. Matis ${ }^{27}$ O. Matonoha,${ }^{13}$ J. A. Mazer, ${ }^{43}$ K. Meehan, ${ }^{7}$ J. C. Mei ${ }^{46}$ N. G. Minaev ${ }^{39}$ S. Mioduszewski ${ }^{51}$ D. Mishra ${ }^{32}$ B. Mohanty, ${ }^{32}$ M. M. Mondal, ${ }^{22}$ I. Mooney, ${ }^{59}$ Z. Moravcova, ${ }^{13}$ D. A. Morozov ${ }^{39}$ Md. Nasim, ${ }^{8}$ K. Nayak,${ }^{10}$ J. M. Nelson, ${ }^{6}$ D. B. Nemes,${ }^{60}$ M. Nie, ${ }^{47}$ G. Nigmatkulov, ${ }^{31}$ T. Niida, ${ }^{59}$ L. V. Nogach, ${ }^{39}$ T. Nonaka, ${ }^{10}$ G. Odyniec, ${ }^{27}$ A. Ogawa, ${ }^{5}$ K. Oh, ${ }^{41}$ S. Oh,${ }^{60}$ V. A. Okorokov, ${ }^{31}$ D. Olvitt, Jr. ${ }^{50}$ B. S. Page,${ }^{5}$ R. Pak, ${ }^{5}$ Y. Panebratsev, ${ }^{24}$ B. Pawlik, ${ }^{36}$ H. Pei, ${ }^{10}$ C. Perkins, ${ }^{6}$ R. L. Pinter, ${ }^{15}$ J. Pluta, ${ }^{58}$ J. Porter ${ }^{27}$ M. Posik, ${ }^{50}$ N. K. Pruthi, ${ }^{37}$ M. Przybycien, ${ }^{2}$ J. Putschke, ${ }^{59}$ A. Quintero, ${ }^{50}$ S. K. Radhakrishnan, ${ }^{27}$ S. Ramachandran, ${ }^{26}$ R. L. Ray, ${ }^{52}$ R. Reed ${ }^{28}$ H. G. Ritter, ${ }^{27}$ J. B. Roberts ${ }^{42}$ O. V. Rogachevskiy, ${ }^{24}$ J. L. Romero, ${ }^{7}$ L. Ruan, ${ }^{5}$ J. Rusnak ${ }^{34}$ O. Rusnakova, ${ }^{13}$ N. R. Sahoo, ${ }^{51}$ P. K. Sahu, ${ }^{22}$ S. Salur, ${ }^{43}$ J. Sandweiss, ${ }^{60}$ J. Schambach, ${ }^{52}$ A. M. Schmah, ${ }^{27}$ W. B. Schmidke, ${ }^{5}$ N. Schmitz ${ }^{29}$ B. R. Schweid ${ }^{49}$ F. Seck ${ }^{14}$ J. Seger, ${ }^{12}$ M. Sergeeva, ${ }^{8}$ R. Seto,${ }^{9}$ P. Seyboth ${ }^{29}$ N. Shah, ${ }^{47}$ E. Shahaliev,${ }^{24}$

P. V. Shanmuganathan, ${ }^{28}$ M. Shao ${ }^{45}$ W. Q. Shen, ${ }^{47}$ S. S. Shi, ${ }^{10}$ Q. Y. Shou,${ }^{47}$ E. P. Sichtermann, ${ }^{27}$ S. Siejka ${ }^{58}$ R. Sikora, ${ }^{2}$ M. Simko, ${ }^{34}$ J. Singh,${ }^{37}$ S. Singha, ${ }^{25}$ D. Smirnov, ${ }^{5}$ N. Smirnov, ${ }^{60}$ W. Solyst,${ }^{21}$ P. Sorensen, ${ }^{5}$ H. M. Spinka, ${ }^{4}$ B. Srivastava, ${ }^{40}$ T. D. S. Stanislaus, ${ }^{56}$ D. J. Stewart, ${ }^{60}$ M. Strikhanov, ${ }^{31}$ B. Stringfellow, ${ }^{40}$ A. A. P. Suaide, ${ }^{44}$ T. Sugiura, ${ }^{54}$ M. Sumbera, ${ }^{34}$ B. Summa, ${ }^{38}$ X. M. Sun,${ }^{10}$ Y. Sun,${ }^{45}$ Y. Sun,${ }^{20}$ B. Surrow,${ }^{50}$ D. N. Svirida,${ }^{3}$ P. Szymanski,${ }^{58}$ A. H. Tang, ${ }^{5}$ Z. Tang,${ }^{45}$ A. Taranenko, ${ }^{31}$ T. Tarnowsky, ${ }^{30}$ J. H. Thomas, ${ }^{27}$ A. R. Timmins, ${ }^{19}$ T. Todoroki, ${ }^{5}$ M. Tokarev,${ }^{24}$ C. A. Tomkiel, ${ }^{28}$ S. Trentalange, ${ }^{8}$ R. E. Tribble, ${ }^{51}$ P. Tribedy, ${ }^{5}$ S. K. Tripathy, ${ }^{22}$ O. D. Tsai, ${ }^{8}$ B. Tu, ${ }^{10}$ T. Ullrich, ${ }^{5}$ D. G. Underwood, ${ }^{4}$ I. Upsal ${ }^{5,46}$ G. Van Buren, ${ }^{5}$ J. Vanek, ${ }^{34}$ A. N. Vasiliev, ${ }^{39}$ I. Vassiliev, ${ }^{16}$ F. Videbæk, ${ }^{5}$ S. Vokal,${ }^{24}$ S. A. Voloshin, ${ }^{59}$ A. Vossen, ${ }^{21}$ F. Wang, ${ }^{40}$ G. Wang, ${ }^{8}$ P. Wang,${ }^{45}$ Y. Wang, ${ }^{10}$ Y. Wang, ${ }^{53}$ J. C. Webb,${ }^{5}$ L. Wen, ${ }^{8}$ G. D. Westfall,,${ }^{30}$ H. Wieman, ${ }^{27}$ S. W. Wissink, ${ }^{21}$ R. Witt, ${ }^{55}$ Y. Wu, ${ }^{25}$ Z. G. Xiao, ${ }^{53}$ G. Xie, ${ }^{11}$ W. Xie, ${ }^{40}$ H. Xu ${ }^{20}$ N. Xu, ${ }^{27}$ Q. H. Xu, ${ }^{46}$ Y. F. Xu ${ }^{47}$ Z. Xu, ${ }^{5}$ C. Yang, ${ }^{46}$ Q. Yang, ${ }^{46}$ S. Yang, ${ }^{5}$ Y. Yang, ${ }^{33}$ Z. Ye, ${ }^{42}$ Z. Ye, ${ }^{11}$ L. Yi, ${ }^{46}$ K. Yip, ${ }^{5}$ I. -K. Yoo, ${ }^{41}$ H. Zbroszczyk ${ }^{58}$ W. Zha, ${ }^{45}$ D. Zhang, ${ }^{10}$ J. Zhang, ${ }^{49}$ L. Zhang, ${ }^{10}$ S. Zhang, ${ }^{45}$ S. Zhang, ${ }^{47}$ X. P. Zhang, ${ }^{53}$ Y. Zhang, ${ }^{45}$ Z. Zhang, ${ }^{47}$ J. Zhao, ${ }^{40}$ C. Zhong, ${ }^{47}$ C. Zhou, ${ }^{47}$ X. Zhu, ${ }^{53}$ Z. Zhu, ${ }^{46}$ M. Zurek, ${ }^{27}$ and M. Zyzak ${ }^{16}$

\section{(STAR Collaboration)}

\author{
${ }^{1}$ Abilene Christian University, Abilene, Texas 79699 \\ ${ }^{2}$ AGH University of Science and Technology, FPACS, Cracow 30-059, Poland \\ ${ }^{3}$ Alikhanov Institute for Theoretical and Experimental Physics, Moscow 117218, Russia \\ ${ }^{4}$ Argonne National Laboratory, Argonne, Illinois 60439 \\ ${ }^{5}$ Brookhaven National Laboratory, Upton, New York 11973
}




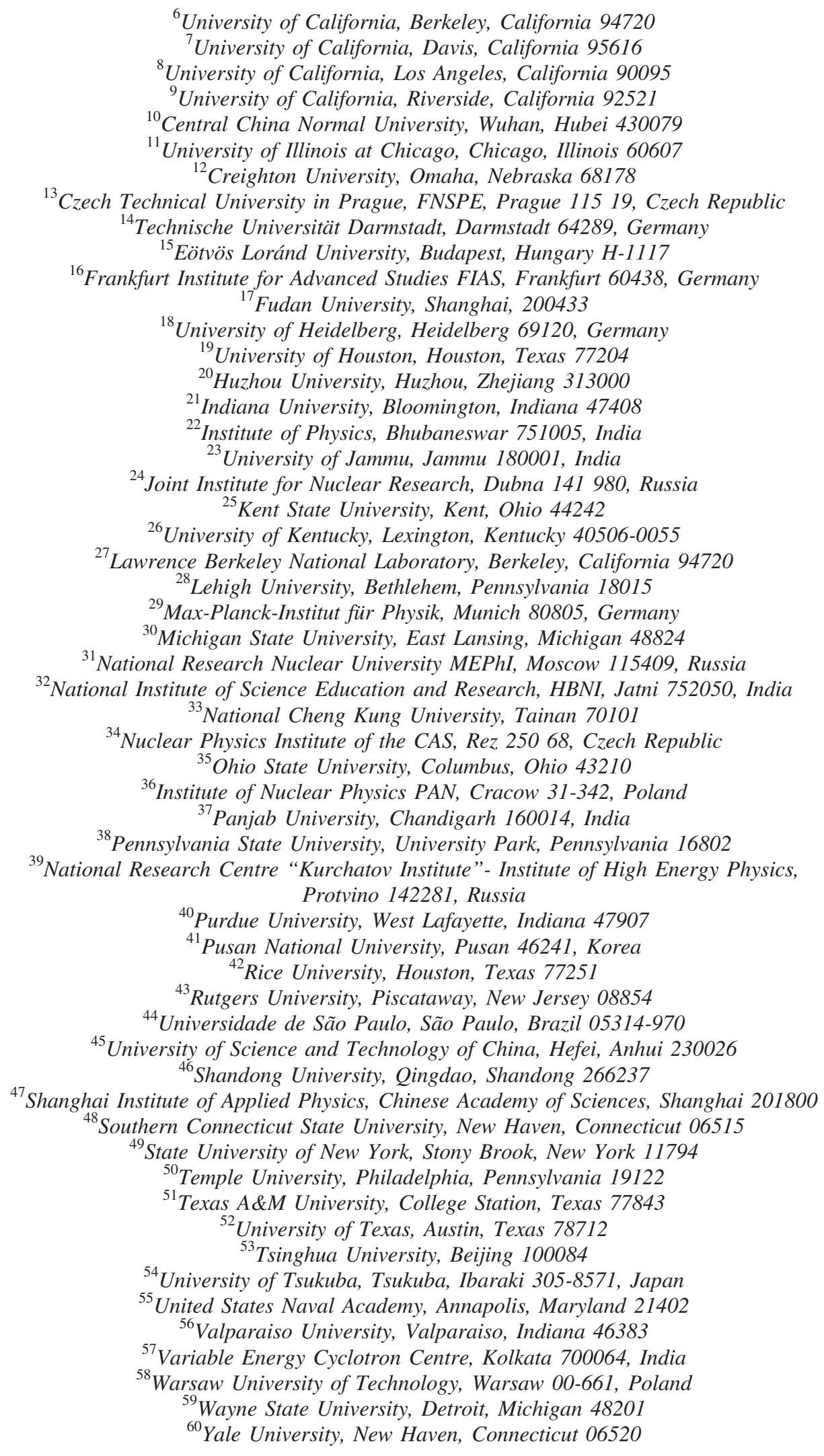


(Received 12 December 2018; published 14 March 2019)

\begin{abstract}
We report new STAR measurements of the single-spin asymmetries $A_{L}$ for $W^{+}$and $W^{-}$bosons produced in polarized proton-proton collisions at $\sqrt{s}=510 \mathrm{GeV}$ as a function of the decay-positron and decayelectron pseudorapidity. The data were obtained in 2013 and correspond to an integrated luminosity of $250 \mathrm{pb}^{-1}$. The results are combined with previous results obtained with $86 \mathrm{pb}^{-1}$. A comparison with theoretical expectations based on polarized lepton-nucleon deep-inelastic scattering and prior polarized proton-proton data suggests a difference between the $\bar{u}$ and $\bar{d}$ quark helicity distributions for $0.05<x<0.25$. In addition, we report new results for the double-spin asymmetries $A_{L L}$ for $W^{ \pm}$, as well as $A_{L}$ for $Z / \gamma^{*}$ production and subsequent decay into electron-positron pairs.
\end{abstract}

DOI: 10.1103/PhysRevD.99.051102

Understanding the spin structure of the proton in terms of its quark, antiquark, and gluon constituents is of fundamental interest. This description is commonly done using polarized parton distribution functions (PDFs), which can be determined using perturbative QCD techniques and global analyses of data from polarized deep-inelastic lepton-nucleon scattering (DIS) experiments and from high-energy polarized proton-proton scattering experiments at the Relativistic Heavy Ion Collider (RHIC). Recent examples of such PDFs are given in Refs. [1,2]. The data from leptonic $W$-decays in polarized protonproton collisions at RHIC [3-7] provide constraints in these global analyses, which now show a flavor asymmetry in the light sea-quark polarizations for parton momentum fractions, $0.05<x<0.25$, at hard perturbative scales. The existence of such an asymmetry in the polarized PDFs has been searched for directly in semi-inclusive DIS experiments [8-10] but had thus far been established only in the case of the unpolarized PDFs. There, Drell-Yan measurements [11,12] and DIS measurements [13,14], in particular, have reported large enhancements in the ratio of $\bar{d}$ over $\bar{u}$ antiquark distributions. This has provided a strong impetus for theoretical modeling [15] and renewed measurement [16]. Considerable progress is being made also in latticeQCD [17].

The leptonic $W^{+} \rightarrow e^{+} \nu$ and $W^{-} \rightarrow e^{-} \bar{\nu}$ decay channels provide sensitivity to the helicity distributions of the quarks, $\Delta u$ and $\Delta d$, and antiquarks, $\Delta \bar{u}$ and $\Delta \bar{d}$, that is free of uncertainties associated with nonperturbative fragmentation. The cross sections are well described [18]. The primary observable is the longitudinal single-spin asymmetry $A_{L} \equiv\left(\sigma_{+}-\sigma_{-}\right) /\left(\sigma_{+}+\sigma_{-}\right)$where $\sigma_{+(-)}$is the cross section when the helicity of the polarized proton beam is positive (negative). At leading order,

Published by the American Physical Society under the terms of the Creative Commons Attribution 4.0 International license. Further distribution of this work must maintain attribution to the author(s) and the published article's title, journal citation, and DOI. Funded by SCOAP ${ }^{3}$.

$$
\begin{aligned}
& A_{L}^{W^{+}}\left(y_{W}\right) \propto \frac{\Delta \bar{d}\left(x_{1}\right) u\left(x_{2}\right)-\Delta u\left(x_{1}\right) \bar{d}\left(x_{2}\right)}{\bar{d}\left(x_{1}\right) u\left(x_{2}\right)+u\left(x_{1}\right) \bar{d}\left(x_{2}\right)}, \\
& A_{L}^{W^{-}}\left(y_{W}\right) \propto \frac{\Delta \bar{u}\left(x_{1}\right) d\left(x_{2}\right)-\Delta d\left(x_{1}\right) \bar{u}\left(x_{2}\right)}{\bar{u}\left(x_{1}\right) d\left(x_{2}\right)+d\left(x_{1}\right) \bar{u}\left(x_{2}\right)},
\end{aligned}
$$

where $x_{1}\left(x_{2}\right)$ is the momentum fraction carried by the colliding quark or antiquark in the polarized (unpolarized) beam. $A_{L}^{W^{+}}\left(A_{L}^{W^{-}}\right)$approaches $-\Delta u / u(-\Delta d / d)$ in the very forward region of $W$ rapidity, $y_{W} \gg 0$, and $\Delta \bar{d} / \bar{d}(\Delta \bar{u} / \bar{u})$ in the very backward region of $W$ rapidity, $y_{W} \ll 0$. The observed positron and electron pseudorapidities, $\eta_{e}$, are related to $y_{W}$ and to the decay angle of the positron and electron in the $W$ rest frame [19]. Higher-order corrections to $A_{L}\left(\eta_{e}\right)$ are known [20-22] and have been incorporated into the aforementioned global analyses.

In this article, we report new measurements of the single-spin asymmetries for decay positrons and electrons from $W^{ \pm}$bosons produced in longitudinally polarized proton-proton collisions at a center-of-mass energy of $\sqrt{s}=510 \mathrm{GeV}$. In addition, we report new results for the double-spin asymmetries $A_{L L}$ for $W^{ \pm}$and $A_{L}$ for $Z / \gamma^{*}$ production. The data were recorded in the year 2013 by the STAR collaboration and correspond to an integrated luminosity of about $250 \mathrm{pb}^{-1}$. The polarizations of the two incident proton beams were measured using Coulombnuclear interference proton-carbon polarimeters, which were calibrated with a polarized hydrogen gas-jet target [23]. The luminosity-weighted beam polarization was $P=0.56$, with a relative scale uncertainty of $3.3 \%$ for the single-beam polarization and $6.4 \%$ for the product of the polarizations from both beams. The figure-of-merit, $P^{2} \mathcal{L}$ for single-spin asymmetry measurements, is higher by a factor of three for the 2013 data compared to the results [4] from the 2011 and 2012 data.

This measurement and analysis made use of essentially the same apparatus and techniques as described in Refs. $[3,4,18]$. As before, the subsystems of the STAR detector [24] used in this measurement are the Time Projection Chamber [25] (TPC), which provides charged particle tracking for pseudorapidities $|\eta| \lesssim 1.3$, and the Barrel [26] and Endcap [27] Electromagnetic Calorimeters 
(BEMC, EEMC). These lead-scintillator sampling calorimeters are segmented into optically isolated towers that cover the full azimuthal angle, $\phi$, for mid and forward pseudorapidity, $|\eta|<1.0$ and $1.1<\eta<2.0$, respectively. They provide the online triggering requirements to initiate the data recording. The trigger accepted events if a transverse energy $E_{T}>12(10) \mathrm{GeV}$ was observed in a region $\Delta \eta \times \Delta \phi \simeq 0.1 \times 0.1$ of the BEMC (EEMC). Events were kept in the analysis if their collision vertex along the beam axis, determined from tracks reconstructed in the TPC, was within $\pm 100 \mathrm{~cm}$ of the center of the STAR detector. The vertex distribution along the beam axis was approximately Gaussian with an RMS width of $47 \mathrm{~cm}$.

The $W^{ \pm}$bosons were detected via their decay into positrons and electrons, $W^{+} \rightarrow e^{+} \nu$ and $W^{-} \rightarrow e^{-} \bar{\nu}$. These events are characterized by an isolated $e^{+}$or $e^{-}$with high transverse momentum, $p_{T}$, accompanied by a high $p_{T}$ neutrino, $\nu$, or antineutrino, $\bar{\nu}$. Since the $\nu$ and $\bar{\nu}$ escape detection, this leads to a characteristically large $p_{T}$ imbalance in these events.

Candidate $W$-decay positrons or electrons were identified at midrapidity (forward rapidity) by a high $p_{T}$ TPC track associated with the primary event collision vertex pointing to a matching tower cluster in the BEMC (EEMC) with high energy. Candidate tracks at midrapidity (forward rapidity) were required to have at least 15 (5) TPC hits to ensure good track quality, and the ratio of the number of hits in the fit to the number of possible hits was required to be more than 0.51 to avoid splitting tracks. A threshold was imposed on the transverse momentum of the particle track, $p_{T}>10(7) \mathrm{GeV} / c$.

Of the four possible $2 \times 2$ calorimeter tower clusters containing the tower that was hit at its front face by the high- $p_{T}$ positron or electron, the cluster with the largest total energy was used to determine the positron or electron transverse energy, $E_{T}^{e}$. This energy was required to exceed $14 \mathrm{GeV}$. The distance between the track and the center position of the tower cluster was required to be less than $7(10) \mathrm{cm}$ at the front face of the BEMC (EEMC).

Unlike background events, signal events have a characteristic isolated transverse energy deposit from the decay positron or electron of about $40 \mathrm{GeV}$, approximately half the $W$ mass, and a large imbalance in the total observed transverse energy as mentioned above. QCD backgrounds were suppressed using selections based on kinematic and topological differences between leptonic $W$-decay events and QCD processes. To identify isolated high- $p_{T}$ decay positrons or electrons, and discriminate against jets, the ratio of $E_{T}^{e}$ to the total energy in a $4 \times 4$ BEMC (EEMC) cluster centered on and including the candidate $2 \times 2$ tower cluster was required to be greater than $95 \%(96 \%)$. In addition, the ratio of $E_{T}^{e}$ to the transverse energy $E_{T}^{\Delta R<0.7}$ in a cone of radius of $\Delta R=\sqrt{\Delta \eta^{2}+\Delta \phi^{2}}<0.7$ around the candidate track was required to be greater than $88 \%$. The transverse energy $E_{T}^{\Delta R<0.7}$ was determined by summing the BEMC and EEMC $E_{T}$ and the TPC track $p_{T}$ within the cone. This selection thus suppressed jet-like events. In addition, in the EEMC acceptance, an isolation cut based on the energy deposited in the two layers of the EEMC Shower Maximum Detector (ESMD) [27] was used. The ESMD can be used to measure the transverse profile of the electromagnetic shower and thereby discriminate between the narrow transverse profile of an isolated (signal) positron or electron shower and the typically wider distribution observed in QCD (background) events. This was done by requiring that the ratio of total energy deposited in ESMD strips within $\pm 1.5 \mathrm{~cm}$ of the central strip pointed to by a TPC track to the energy deposited in strips within $\pm 10 \mathrm{~cm}$, $R_{\mathrm{ESMD}}$, was greater than 0.7 .

In addition, the characteristic transverse energy imbalance of signal events was used to further suppress backgrounds. A $p_{T}$-balance vector, $\vec{p}_{T}^{\text {bal }}$, defined as the vector-sum of the decay positron or electron candidate $\vec{p}_{T}^{e}$ vector plus the sum of the $\vec{p}_{T}$ vectors for all reconstructed jets whose axes are outside a cone radius of $\Delta R=0.7$ around the candidate decay positron or electron, was computed for each event. Jets were reconstructed for this purpose using an anti- $k_{T}$ algorithm [28] with a resolution parameter $R=0.6$ from towers (tracks) with $E_{T}\left(p_{T}\right)>0.2 \mathrm{GeV}(/ c)$. Reconstructed jets were required to have $p_{T}>3.5 \mathrm{GeV} / c$. A scalar signed $p_{T^{-}}$ balance variable, defined as $\left(\vec{p}_{T}^{e} \cdot \vec{p}_{T}^{\text {bal }}\right) /\left|\vec{p}_{T}^{e}\right|$, was then computed and required to be larger than 14 (20) GeV/c for candidate events in the BEMC (EEMC) to be retained in the analysis. Complementary to the signed $p_{T}$-balance cut, it was required that the total transverse energy opposite in azimuth to the candidate positron or electron in the BEMC, $-0.7<\Delta \phi-\pi<0.7$, did not exceed $11 \mathrm{GeV}$. This further reduced QCD dijet background in cases when a sizable fraction of the energy for one of the jets was not observed due to detector effects.

Candidate positrons or electrons that passed the above selection cuts were then sorted by charge-sign, determined from the curvature of the TPC tracks in the solenoidal magnetic field. Figure 1(a) and 1(b) show the distribution of the reconstructed charge-sign, $Q= \pm 1$, multiplied by
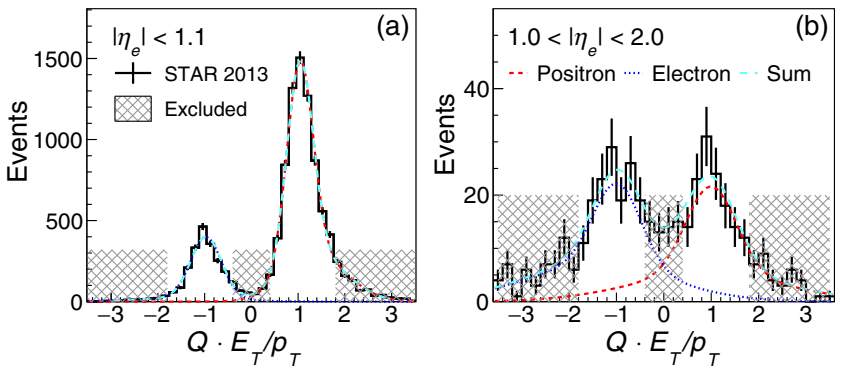

FIG. 1. Distribution of the product of $Q$, the TPC reconstructed charge-sign, and $E_{T} / p_{T}$ in the BEMC (a) and EEMC (b) regions. The positron (red) and electron (blue) candidate events have been fitted with double-Gaussian distributions. The excluded regions are marked by hatched shades. 
the ratio of $E_{T}^{e}$ observed in the BEMC and EEMC to $p_{T}^{e}$ determined with the TPC for events in the signal region, $25<E_{T}^{e}<50 \mathrm{GeV}$. The relative yields of the $W^{+}$and $W^{-}$ follow the pseudorapidity dependence of the cross-section ratio. The distributions were each fitted with two doubleGaussian template shapes, determined from a Monte Carlo simulated $W$ sample, to estimate the reconstructed chargesign purity. The amplitudes of the Gaussians were fitted to the data, as was the central position of the narrower Gaussian in each of the templates. The remaining parameters were fixed by studies in which simulated $W^{+} \rightarrow e^{+} \nu$ and $W^{-} \rightarrow e^{-} \bar{\nu}$ events were embedded (c.f. the paragraph below) in zero-bias data. The hatched regions, $\left|Q \cdot E_{T} / p_{T}\right|<0.4$ and $\left|Q \cdot E_{T} / p_{T}\right|>1.8$, were excluded to remove tracks with poorly reconstructed $p_{T}$ and to reduce contamination from events with opposite chargesign. This contamination is negligible at midrapidity, but increases to $9.6 \%$ and $12.0 \%$ for $W^{+}$and $W^{-}$candidate events, respectively, in the EEMC region. The forward $A_{L}$ values were corrected for this contamination using the asymmetries observed in the data.

Figure 2 shows the distributions of $W^{+}$and $W^{-}$yields as a function of $E_{T}^{e}$ for the four central $\eta_{e}$ intervals considered in this analysis, along with the estimated residual background contributions from electroweak and QCD processes. The residual electroweak backgrounds are predominantly due to $W^{ \pm} \rightarrow \tau^{ \pm} \nu_{\tau}$ and $Z / \gamma^{*} \rightarrow e^{+} e^{-}$. These contributions were estimated from Monte Carlo simulations, using events generated with PYTHIA 6.4.28 [29] and the "Perugia 0" tune [30] that passed through a GEANT 3 [31] model of the STAR detector, and were subsequently embedded into STAR zero-bias data. The simulated samples were normalized to the $W$ data using the known integrated luminosity. The TAUOLA package was used for the polarized $\tau^{ \pm}$decay [32]. Residual QCD dijet background in which one of the jets pointed to uninstrumented pseudorapidity regions was estimated using two separate procedures. The contribution from $e^{ \pm}$candidate events with an opposite-side jet fragment in the uninstrumented region $-2<\eta<-1.1$ was estimated by studying such data in the EEMC, which instruments the region $1.1<\eta<2$. This is referred to as the "Second EEMC" procedure. Residual background from the uninstrumented region $|\eta|>2$ was estimated by studying events that satisfy all isolation criteria, but do not satisfy the cuts on the scalar signed $p_{T}$-balance variable. This is referred to as the "Data-driven QCD" procedure. To assess the background remaining in the signal region, the $E_{T}$ distribution of this background-dominated sample was normalized to the signal candidate distribution that remained after all other background contributions had been removed for $E_{T}$ values between 14 and $18 \mathrm{GeV}$. Additional aspects of both procedures are described in Refs. [3,18].

Figure 3 shows the charge-separated distributions in the EEMC region as a function of the signed $p_{T}$-balance variable, together with the estimated residual background contributions. Residual electroweak backgrounds for these regions were estimated in the same way as for the midrapidity data. Residual QCD backgrounds were estimated using the ESMD, where the isolation parameter $R_{\text {ESMD }}$ was required to be less than 0.6 for QCD background events. The shape was determined for each chargesign separately and normalized to the measured yield in the region where the signed $p_{T}$-balance variable was between -8 and $8 \mathrm{GeV} / c$. This region is dominated by QCD backgrounds.

At RHIC, there are four helicity configurations for the two longitudinally-polarized proton beams:,,+++--+ ,

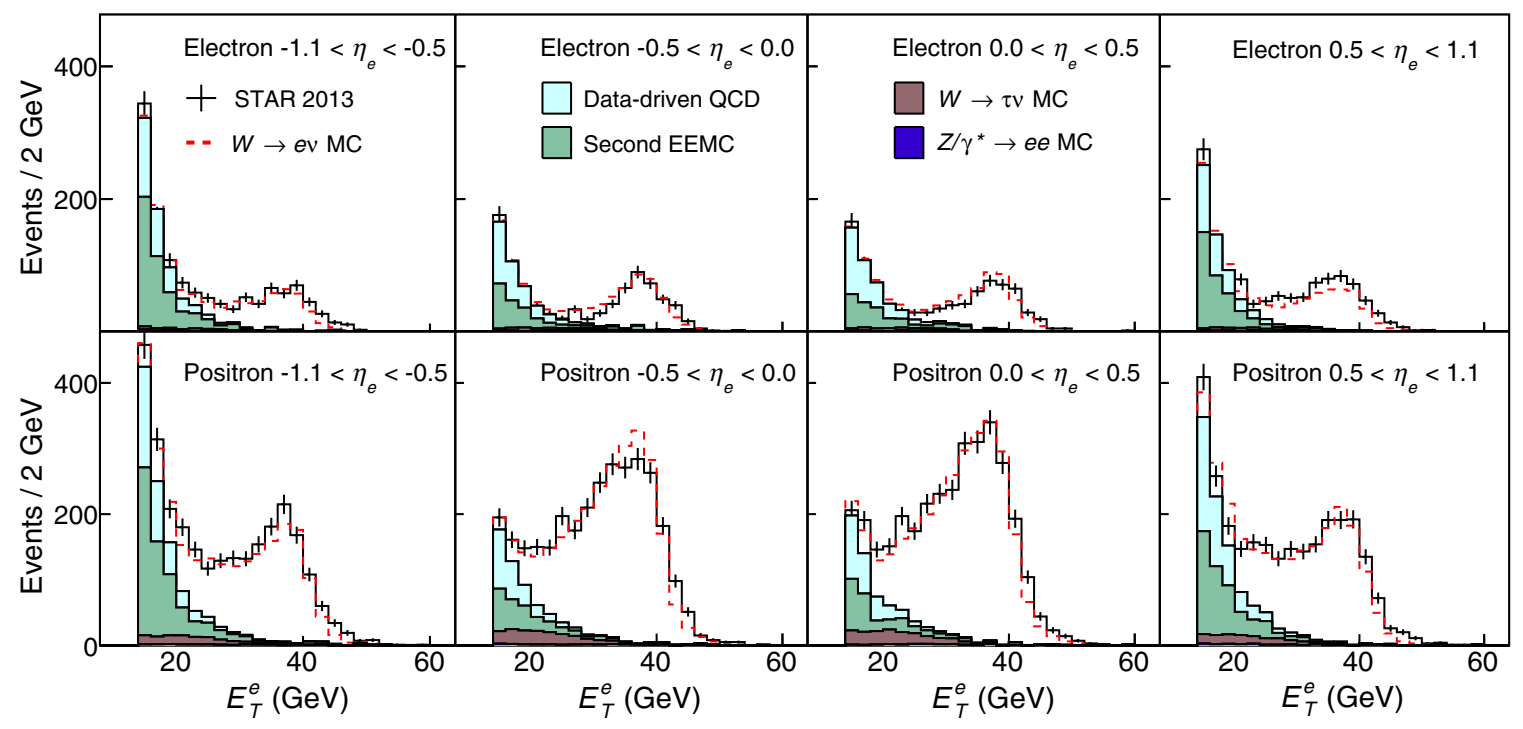

FIG. 2. $\quad E_{T}^{e}$ distribution of electron (top) and positron (bottom) candidates (black crosses), background contributions, and sum of backgrounds and $W \rightarrow e \nu$ MC signal (red-dashed) in the BEMC region. 


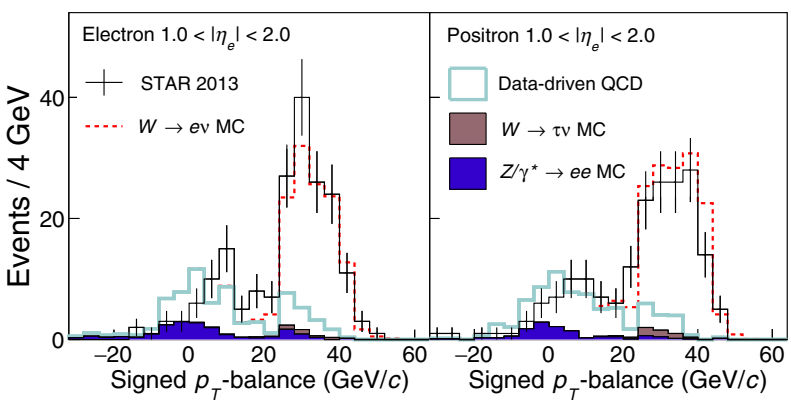

FIG. 3. Signed $p_{T}$-balance distribution for electron (left) and positron (right) candidates (black crosses), background contributions, and sum of backgrounds and $W \rightarrow e \nu$ MC signal (reddashed) in the EEMC region.

and --. The data from these four configurations can be combined such that the net polarization for one beam effectively averages to zero, while maintaining high polarization in the other. The longitudinal single-spin asymmetry $A_{L}$ for the combination in which the first beam is polarized and the second carries no net polarization was determined from

$A_{L}=\frac{1}{\beta P} \frac{R_{++} N_{++}+R_{+-} N_{+-}-R_{-+} N_{-+}-R_{--} N_{--}}{R_{++} N_{++}+R_{+-} N_{+-}+R_{-+} N_{-+}+R_{--} N_{--}}$,

where $\beta$ is the signal purity, $P$ is the average beam polarization, and $R$ and $N$ are the normalizations for relative luminosity and the raw $W^{ \pm}$yields, respectively, for the helicity configurations indicated by the subscripts. The relative luminosities were obtained from a large QCD sample that exhibits no significant single-spin asymmetry. Typical values were between 0.993 and 1.009 . The purity was evaluated from the aforementioned signal and background contributions and was found to be between $83 \%$ and $98 \% . A_{L}$ was determined in a similar way for the combination in which the second beam is polarized and the first carries no net polarization, and the values for the two combinations were then combined.

The $A_{L}$ results for $W^{+}$and $W^{-}$from the data sample recorded by STAR in 2013 are shown in Fig. 4 as a function of $\eta_{e}$. The vertical error bars show the size of the statistical uncertainties, including those associated with the correction for the wrong charge-sign in the case of the points at $\left|\eta_{e}\right| \simeq 1.2$. The previously published STAR data [4] are shown for comparison. Shown also are the $A_{L}$ data on high-energy forward decay muons and midrapidity positrons or electrons from combined $W$ and $Z / \gamma^{*}$ production by the PHENIX experiment with their statistical and systematic uncertainties as a function of $\eta_{\mu}$ and $\eta_{e}$, respectively $[6,7]$.

The size of systematic uncertainties associated with BEMC and EEMC gain calibrations (5\% variation) and the data-driven QCD background are indicated by the boxes.

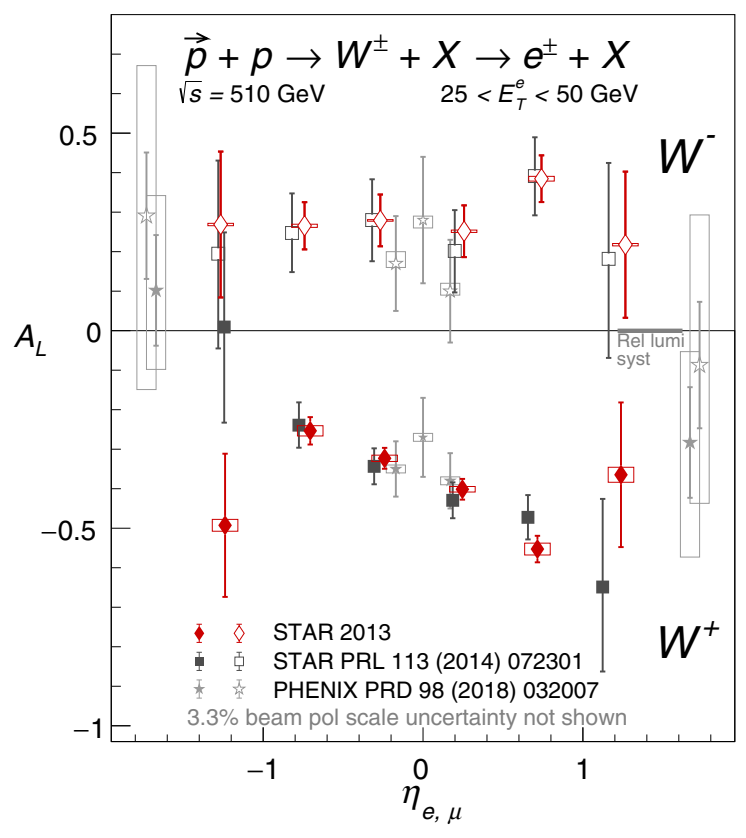

FIG. 4. Longitudinal single-spin asymmetries, $A_{L}$, for $W^{ \pm}$ production as a function of the positron or electron pseudorapidity, $\eta_{e}$, separately for the STAR $2011+2012$ (black squares) and 2013 (red diamonds) data samples for $25<E_{T}^{e}<50 \mathrm{GeV}$. The $2011+2012$ results have been offset to slightly smaller $\eta$ values for clarity. Shown also are the final asymmetries for highenergy decay leptons from $W$ and $Z / \gamma^{*}$ production from the PHENIX central arms as a function of $\eta_{e}$ and from the muon-arms as a function of $\eta_{\mu}$ with their statistical and systematic uncertainties [6,7].

The gray band shown along the $A_{L}=0$ line indicates the size of the systematic uncertainty from the determination of relative luminosity, and is correlated among all the points. The $3.3 \%$ relative systematic uncertainty from beam polarization measurement is not shown. Table I gives the results for $A_{L}$, as well as for the longitudinal double-spin asymmetry $A_{L L} \equiv\left(\sigma_{++}+\sigma_{--}-\sigma_{+-}-\sigma_{-+}\right) /\left(\sigma_{++}+\sigma_{--}+\sigma_{+-}+\sigma_{-+}\right)$, where the subscripts denote the helicity configurations. The new $W^{ \pm} A_{L L}$ data are consistent with previously published STAR data [4] and have better precision. $W^{ \pm} A_{L L}$ is sensitive to quark and antiquark polarizations, albeit less so than $A_{L}$, and has been proposed for tests of consistency and positivity constraints $[33,34]$.

The new $W^{ \pm} A_{L}$ data are consistent with the previously published results, and have statistical uncertainties that are 40\%-50\% smaller. The combined STAR data are shown in Fig. 5 and compared with expectations based on the DSSV14 [2], NNPDFpol1.1 [1] and BS15 [35] PDFs evaluated using the next-to-leading order CHE [21] and fully resummed RHICBOS [22] codes. The NNPDFpol1.1 analysis, unlike DSSV14 and BS15, includes the STAR $2011+2012 W^{ \pm}$data [4], which reduces in particular the uncertainties for $W^{-}$expectations at negative $\eta$. To assess the impact, the STAR 2013 data were used in the 
TABLE I. Longitudinal single- and double-spin asymmetries, $A_{L}$ and $A_{L L}$, for $W^{ \pm}$production obtained from the STAR 2013 data sample, as well as the combination of 2013 with $2011+2012$ results. The longitudinal single-spin asymmetry is measured for six decay positron or electron pseudorapidity intervals. The longitudinal double-spin asymmetry was determined in the same intervals and the results for the same absolute pseudorapidity value were combined. The systematic uncertainties include all contributions and thus also include the point-by-point correlated uncertainties from the relative luminosity and beam polarization measurements that are broken out separately in Figs. 4 and 5.

\begin{tabular}{|c|c|c|c|c|c|}
\hline & \multirow[b]{2}{*}{$\left\langle\eta_{e}\right\rangle$} & \multicolumn{2}{|c|}{$A_{L} \pm \sigma_{\text {stat }} \pm \sigma_{\text {syst }}$} & \multicolumn{2}{|c|}{$A_{L L} \pm \sigma_{\text {stat }} \pm \sigma_{\text {syst }}$} \\
\hline & & 2013 & 2011-2013 & 2013 & 2011-2013 \\
\hline \multirow{6}{*}{$W^{+}$} & -1.24 & $-0.493 \pm 0.181 \pm 0.022$ & $-0.312 \pm 0.145 \pm 0.017$ & & \\
\hline & -0.72 & $-0.255 \pm 0.035 \pm 0.016$ & $-0.251 \pm 0.030 \pm 0.014$ & $\ldots$ & $\cdots$ \\
\hline & -0.25 & $-0.327 \pm 0.027 \pm 0.014$ & $-0.331 \pm 0.023 \pm 0.014$ & & \\
\hline & 0.25 & $-0.406 \pm 0.027 \pm 0.016$ & $-0.412 \pm 0.023 \pm 0.016$ & $0.039 \pm 0.049 \pm 0.014$ & $0.016 \pm 0.042 \pm 0.011$ \\
\hline & 0.72 & $-0.557 \pm 0.034 \pm 0.024$ & $-0.534 \pm 0.029 \pm 0.022$ & $0.049 \pm 0.063 \pm 0.014$ & $0.072 \pm 0.054 \pm 0.011$ \\
\hline & 1.24 & $-0.365 \pm 0.183 \pm 0.023$ & $-0.482 \pm 0.140 \pm 0.022$ & $-0.052 \pm 0.331 \pm 0.044$ & $0.000 \pm 0.262 \pm 0.028$ \\
\hline \multirow{6}{*}{$W^{-}$} & -1.27 & $0.269 \pm 0.185 \pm 0.010$ & $0.241 \pm 0.146 \pm 0.010$ & & \\
\hline & -0.74 & $0.264 \pm 0.060 \pm 0.010$ & $0.260 \pm 0.051 \pm 0.010$ & $\cdots$ & $\cdots$ \\
\hline & -0.27 & $0.282 \pm 0.066 \pm 0.010$ & $0.281 \pm 0.056 \pm 0.011$ & & \\
\hline & 0.27 & $0.254 \pm 0.066 \pm 0.010$ & $0.239 \pm 0.056 \pm 0.010$ & $0.067 \pm 0.120 \pm 0.025$ & $-0.012 \pm 0.101 \pm 0.019$ \\
\hline & 0.74 & $0.383 \pm 0.059 \pm 0.015$ & $0.385 \pm 0.051 \pm 0.014$ & $-0.096 \pm 0.107 \pm 0.026$ & $-0.028 \pm 0.092 \pm 0.020$ \\
\hline & 1.27 & $0.218 \pm 0.185 \pm 0.009$ & $0.205 \pm 0.148 \pm 0.009$ & $-0.133 \pm 0.331 \pm 0.061$ & $-0.147 \pm 0.260 \pm 0.038$ \\
\hline
\end{tabular}

reweighting procedure of Refs. [36,37] with the 100 publicly available NNPDFpol1.1 PDFs. The results from this reweighting, taking into account the total uncertainties of the STAR 2013 data and their correlations [38], are shown in Fig. 5 as the blue hatched bands. The NNPDFpol1.1 uncertainties [1] are shown as the green

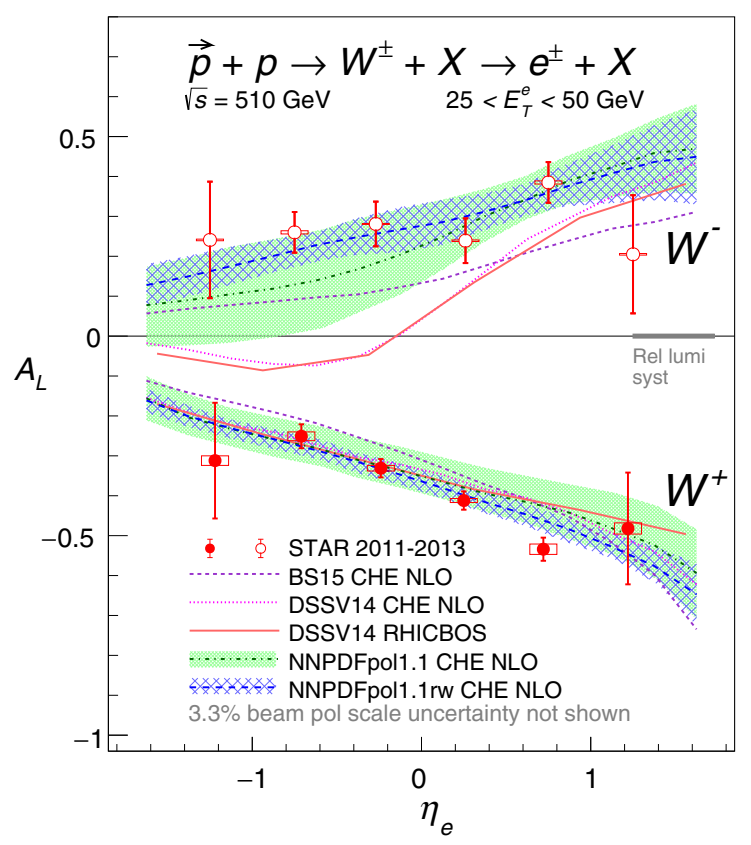

FIG. 5. Longitudinal single-spin asymmetries, $A_{L}$, for $W^{ \pm}$ production as a function of the positron or electron pseudorapidity, $\eta_{e}$, for the combined STAR $2011+2012$ and 2013 data samples for $25<E_{T}^{e}<50 \mathrm{GeV}$ (points) in comparison to theory expectations (curves and bands) described in the text. bands for comparison. Figure 6 shows the corresponding differences of the light sea-quark polarizations versus $x$ at a scale of $Q^{2}=10(\mathrm{GeV} / c)^{2}$. The data confirm the existence of a sizable, positive $\Delta \bar{u}$ in the range $0.05<x<0.25$ [4] and the existence of a flavor asymmetry in the polarized quark sea.

In addition, $A_{L}$ was determined for $Z / \gamma^{*}$ production from a sample of 274 electron-positron pairs with $70<m_{e^{+} e^{-}}<110 \mathrm{GeV} / c^{2}$. The $e^{+}$and $e^{-}$were each required to be isolated, have $\left|\eta_{e}\right|<1.1$, and $E_{T}^{e}>14 \mathrm{GeV}$. The result, $A_{L}^{Z / \gamma^{*}}=-0.04 \pm 0.07$, is consistent with that in Ref. [4] but with half the statistical uncertainty.

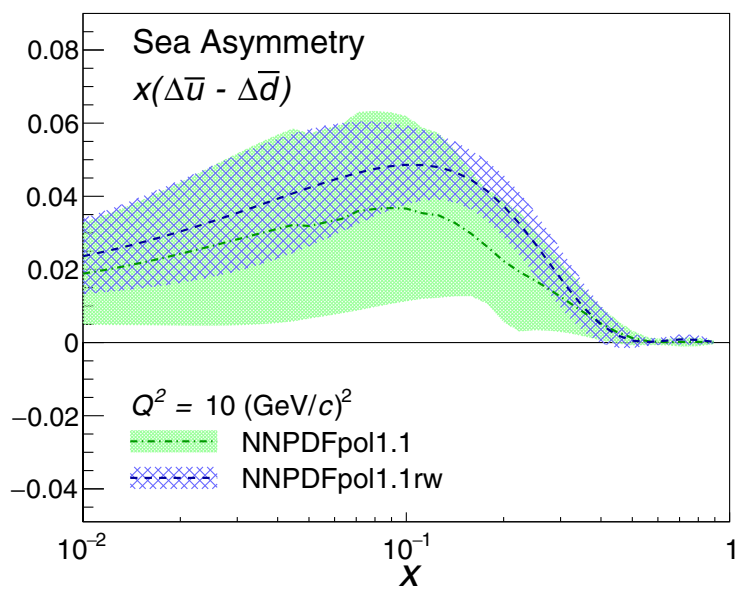

FIG. 6. The difference of the light sea-quark polarizations as a function of $x$ at a scale of $Q^{2}=10(\mathrm{GeV} / c)^{2}$. The green band shows the NNPDFpol1.1 results [1] and the blue hatched band shows the corresponding distribution after the STAR $2013 W^{ \pm}$ data are included by reweighting. 
The systematic uncertainty is negligible compared to the statistical uncertainty. This result is also consistent with theoretical expectations, $A_{L}^{Z / \gamma^{*}}=-0.08$ from DSSV14 [2] and $A_{L}^{Z / \gamma^{*}}=-0.04$ from NNPDFpol1.1 [1].

In summary, we report new STAR measurements of longitudinal single-spin and double-spin asymmetries for $W^{ \pm}$and single-spin asymmetry for $Z / \gamma^{*}$ bosons produced in polarized proton-proton collisions at $\sqrt{s}=510 \mathrm{GeV}$. The production of weak bosons in these collisions and their subsequent leptonic decay is a unique process to delineate the quark and antiquark polarizations in the proton by flavor. The $A_{L}$ data for $W^{+}$and $W^{-}$, combined with previously published STAR results, show a significant preference for $\Delta \bar{u}\left(x, Q^{2}\right)>\Delta \bar{d}\left(x, Q^{2}\right)$ in the fractional momentum range $0.05<x<0.25$ at a scale of $Q^{2}=10(\mathrm{GeV} / c)^{2}$. This is opposite to the flavor asymmetry observed in the spin-averaged quark-sea distributions.
We thank the RHIC Operations Group and RCF at BNL, the NERSC Center at LBNL, and the Open Science Grid consortium for providing resources and support. This work was supported in part by the Office of Nuclear Physics within the U.S. Department of Energy Office of Science, the U.S. National Science Foundation, the Ministry of High Education and Science of the Russian Federation, National Natural Science Foundation of China, Chinese Academy of Science, the Ministry of Science and Technology of China and the Chinese Ministry of Education, the National Research Foundation of Korea, Czech Science Foundation and Ministry of Education, Youth and Sports of the Czech Republic, Department of Atomic Energy and Department of Science and Technology of the Government of India, the National Science Centre of Poland, the Ministry of Science, Education and Sports of the Republic of Croatia, RosAtom of Russia and German Bundesministerium für Bildung, Wissenschaft, Forschung and Technologie (BMBF) and the Helmholtz Association.
[1] E. R. Nocera, R. D. Ball, S. Forte, G. Ridolfi, and J. Rojo (NNPDF Collaboration), Nucl. Phys. B887, 276 (2014).

[2] D. de Florian, R. Sassot, M. Stratmann, and W. Vogelsang, Phys. Rev. Lett. 113, 012001 (2014).

[3] M. M. Aggarwal et al. (STAR Collaboration), Phys. Rev. Lett. 106, 062002 (2011).

[4] L. Adamczyk et al. (STAR Collaboration), Phys. Rev. Lett. 113, 072301 (2014).

[5] A. Adare et al. (PHENIX Collaboration), Phys. Rev. Lett. 106, 062001 (2011).

[6] A. Adare et al. (PHENIX Collaboration), Phys. Rev. D 93, 051103 (2016).

[7] A. Adare et al. (PHENIX Collaboration), Phys. Rev. D 98, 032007 (2018).

[8] B. Adeva et al. (Spin Muon Collaboration), Phys. Lett. B 420, 180 (1998).

[9] A. Airapetian et al. (HERMES Collaboration), Phys. Rev. D 71, 012003 (2005).

[10] M. G. Alekseev et al. (COMPASS Collaboration), Phys. Lett. B 693, 227 (2010).

[11] A. Baldit et al. (NA51 Collaboration), Phys. Lett. B 332, 244 (1994).

[12] R. S. Towell et al. (NuSea Collaboration), Phys. Rev. D 64, 052002 (2001).

[13] M. Arneodo et al. (New Muon Collaboration), Nucl. Phys. B483, 3 (1997).

[14] K. Ackerstaff et al. (HERMES Collaboration), Phys. Rev. Lett. 81, 5519 (1998).

[15] S. Kumano, Phys. Rep. 303, 183 (1998) and references therein.

[16] P. E. Reimer (Fermilab SeaQuest Collaboration), J. Phys. Conf. Ser. 295, 012011 (2011).
[17] H. W. Lin et al., Prog. Part. Nucl. Phys. 100, 107 (2018) and references therein.

[18] L. Adamczyk et al. (STAR Collaboration), Phys. Rev. D 85, 092010 (2012).

[19] G. Bunce, N. Saito, J. Soffer, and W. Vogelsang, Annu. Rev. Nucl. Part. Sci. 50, 525 (2000).

[20] F. Ringer and W. Vogelsang, Phys. Rev. D 91, 094033 (2015).

[21] D. de Florian and W. Vogelsang, Phys. Rev. D 81, 094020 (2010).

[22] P. M. Nadolsky and C. Yuan, Nucl. Phys. B666, 31 (2003).

[23] RHIC Polarimetry Group, The RHIC Polarimetry Group, BNL-209057-2018-TECH, C-A/AP/609 (2018), https:// public.bnl.gov/docs/cad/Documents/RHIC\%20polarization \%20for\%20Runs\%209-17.pdf.

[24] K. H. Ackermann et al., Nucl. Instrum. Methods Phys. Res., Sect. A 499, 624 (2003).

[25] M. Anderson et al., Nucl. Instrum. Methods Phys. Res., Sect. A 499, 659 (2003).

[26] M. Beddo et al., Nucl. Instrum. Methods Phys. Res., Sect. A 499, 725 (2003).

[27] C. E. Allgower et al., Nucl. Instrum. Methods Phys. Res., Sect. A 499, 740 (2003).

[28] M. Cacciari, G. P. Salam, and G. Soyez, J. High Energy Phys. 04 (2008) 063.

[29] T. Sjostrand, S. Mrenna, and P. Z. Skands, J. High Energy Phys. 05 (2006) 026.

[30] P. Z. Skands, Phys. Rev. D 82, 074018 (2010).

[31] R. Brun et al., GEANT: Detector Description and Simulation Tool, CERN Program Library; W5013 (CERN, Geneva, 1993), p. 430.

[32] P. Golonka, B. Kersevan, T. Pierzchała, E. Richter-Wąs, Z. Wąs, and M. Worek, Comput. Phys. Commun. 174, 818 (2006). 
[33] W. C. Chang and J. C. Peng, Prog. Part. Nucl. Phys. 79, 95 (2014).

[34] Z.-B. Kang and J. Soffer, Phys. Rev. D 83, 114020 (2011).

[35] C. Bourrely and J. Soffer, Nucl. Phys. A941, 307 (2015).

[36] R. D. Ball, V. Bertone, F. Cerutti, L. Del Debbio, S. Forte, A. Guffanti, J. I. Latorre, J. Rojo, and M. Ubiali (NNPDF
Collaboration), Nucl. Phys. B849, 112 (2011); 854, 926(E) (2012); 855, 927(E) (2012).

[37] R. D. Ball, V. Bertone, F. Cerutti, L. Del Debbio, S. Forte, A. Guffanti, N. P. Hartland, J. I. Latorre, J. Rojo, and M. Ubiali (NNPDF Collaboration), Nucl. Phys. B855, 608 (2012).

[38] See Supplemental Material at http://link.aps.org/ supplemental/10.1103/PhysRevD.99.051102, for the correlation matrix. 